\title{
Magnetic beads-assisted endoscopic submucosal dissection of duodenal heterotopic gastric mucosa with fibrosis
}

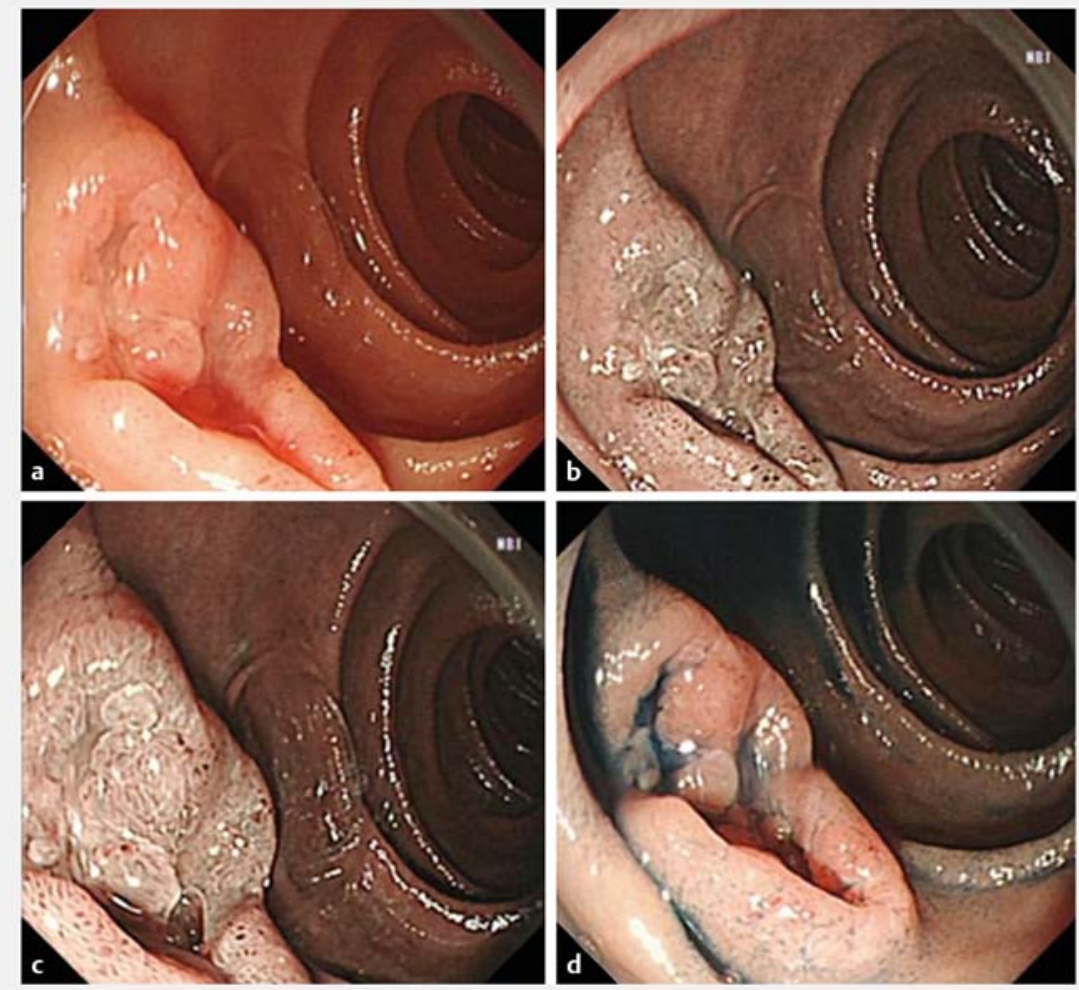

- Fig. 1 Heterotopic gastric mucosa in the duodenum with the appearance of a laterally spreading tumor: a white light appearance; $\mathbf{b}$ narrow band imaging; $\mathbf{c}$ closer look using narrow band imaging; $\mathbf{d}$ indigo carmine staining.

A 53-year-old man was referred to our hospital for full evaluation of the nature of a lesion in the descending duodenum. Upper endoscopy showed a wide-based laterally spreading tumor-like mass (about $15 \times 20 \mathrm{~mm}$ ) with a depression at the top of the lesion ( $\mathbf{F i g} \mathbf{1}$ ). Endoscopic ultrasound (EUS) revealed a medium-echo lesion in the mucosal layer ( Fig. 2). The biopsy result was inconclusive. Diagnostic endoscopic submucosal dissection (ESD) was attempted. However, despite repeated injections, lifting of the lesion was not satisfactory and the submucosal layer could not be exposed clearly, because of fibrosis ( $\triangleright$ Fig.3a). Magnetic beads were used to apply traction by gravity. An endoclip was used to attach the thread of a magnetic bead to the edge of the mucosa. When two magnetic beads had been attached, the cutting line was clearly exposed and the lesion was smoothly dissected en bloc (> Fig.3b-d, - Video 1).

Histological examination demonstrated the presence of pyloric glands and these glands were positive for MUC5AC and MUC6 (\$ Fig. 4). The diagnosis of heterotopic gastric mucosa in the descending duodenum was made, and the patient was discharged uneventfully in 5 days. At 1-month follow-up, the ulcer bed after ESD was seen to be healed ( $>$ Fig.5).

Heterotopic gastric mucosa in the duodenum can mimic the appearance of a polyps, laterally spreading tumor, sub-

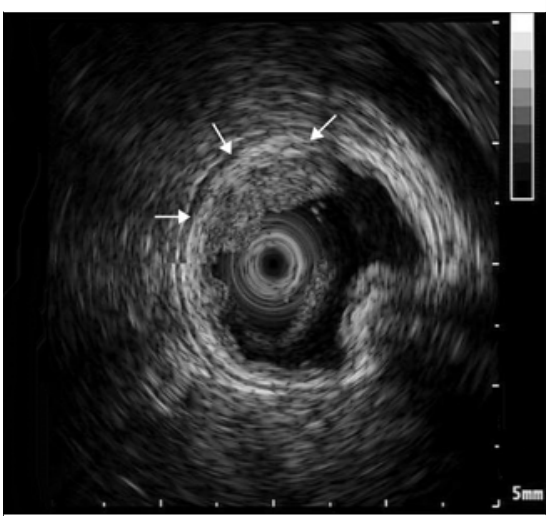

Fig. 2 Endoscopic ultrasound (EUS) revealed a medium-echo lesion (arrows) in the mucosal layer.

mucosal tumor, and so on [1]. In certain cases, ESD is indicated in order to achieve a definitive diagnosis especially when the heterotopic gastric mucosa is large in size. Magnetic beads are a novel traction method that can assist ESD [2].

Endoscopy_UCTN_Code_TTT_1AO_2AG

\section{Competing interests}

All authors have no financial relationships relevant to this article or conflicts of interest to disclose.

\section{The authors}

Lin Jie Guo ${ }^{1,{ }^{*}}$, Liansong $\mathrm{Ye}^{1,{ }^{*}}$, Zhi Ying Huang ${ }^{1}$, Xiaoxue Yin ${ }^{2}$, Bing $\mathrm{Hu}^{1}$

1 Department of Gastroenterology, West China Hospital, Sichuan University, China

2 Department of Pathology, West China Hospital, Sichuan University, China

\footnotetext{
* These authors contributed equally.
} 

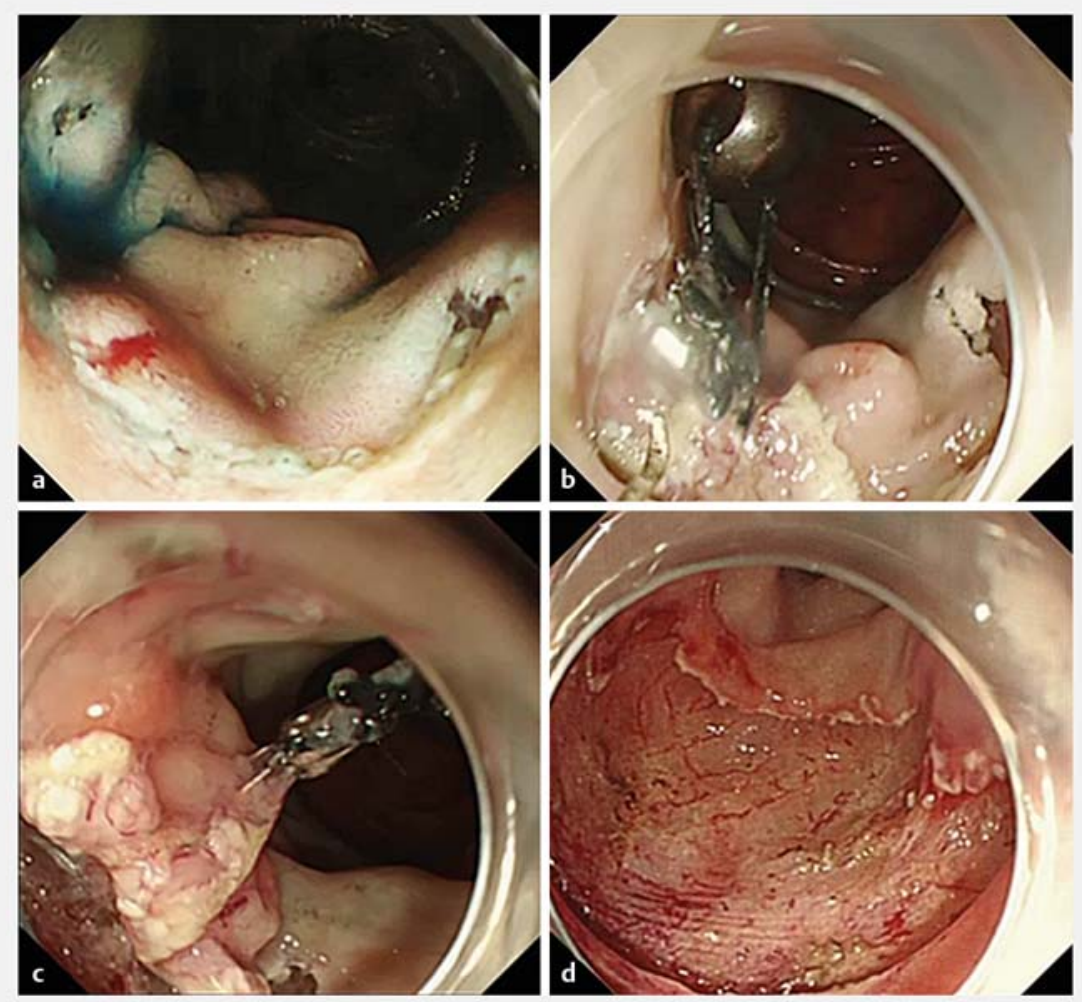

- Fig. 3 Magnetic bead-assisted endoscopic submucosal dissection (ESD). a The submucosal layer could not be clearly exposed despite repeated injections. b Attachment of one magnetic bead to the edge of the mucosa assisted traction by gravity. $\mathbf{c}$ A second magnetic bead was then attached for further traction. $\mathbf{d}$ The ulcer bed after resection.
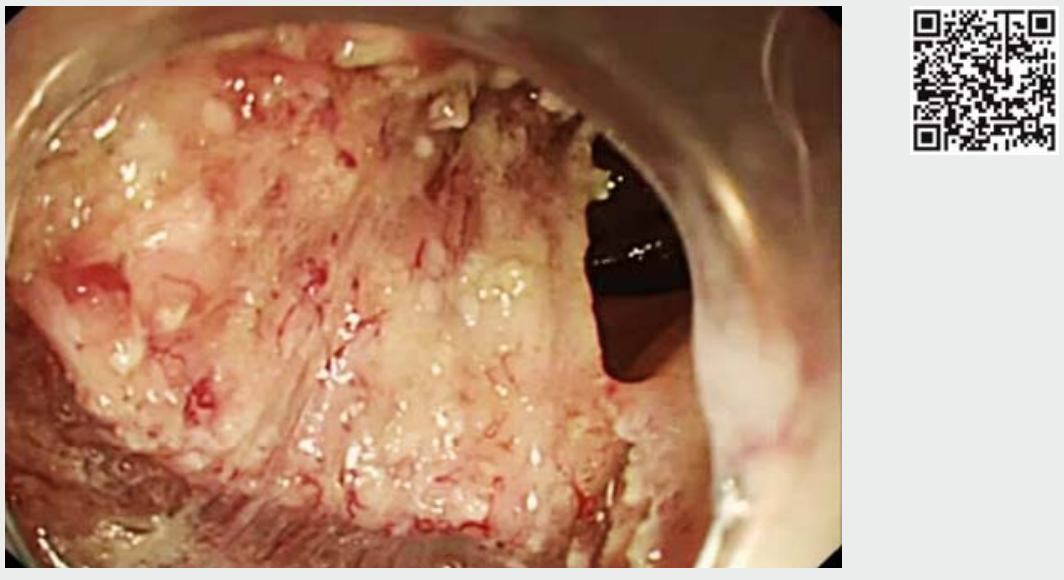

Video 1 Magnetic beads-assisted endoscopic submucosal dissection of duodenal heterotopic gastric mucosa with fibrosis.

\section{Corresponding author}

\section{Bing Hu, MD}

Department of Gastroenterology, West China Hospital, 37 Guo Xue Xiang, Chengdu 610041, Sichuan, China

Fax: +86-028-85423387

hubingnj@163.com

\section{References}

[1] Eguchi K, Aoyagi K, Nimura S et al. Diagnostic value of endoscopic and endoscopic ultrasound characteristics of duodenal submucosal tumour-like heterotopic gastric mucosa. Can J Gastroenterol 2011; 25: 365-367

[2] Bethge J, Ye L, Ellrichmann M et al. Advanced endoscopic submucosal dissection with magnetic bead-assisted traction based on gravity for a flat colorectal neoplasm with severe fibrosis. Endoscopy 2018; 50: 824 825 

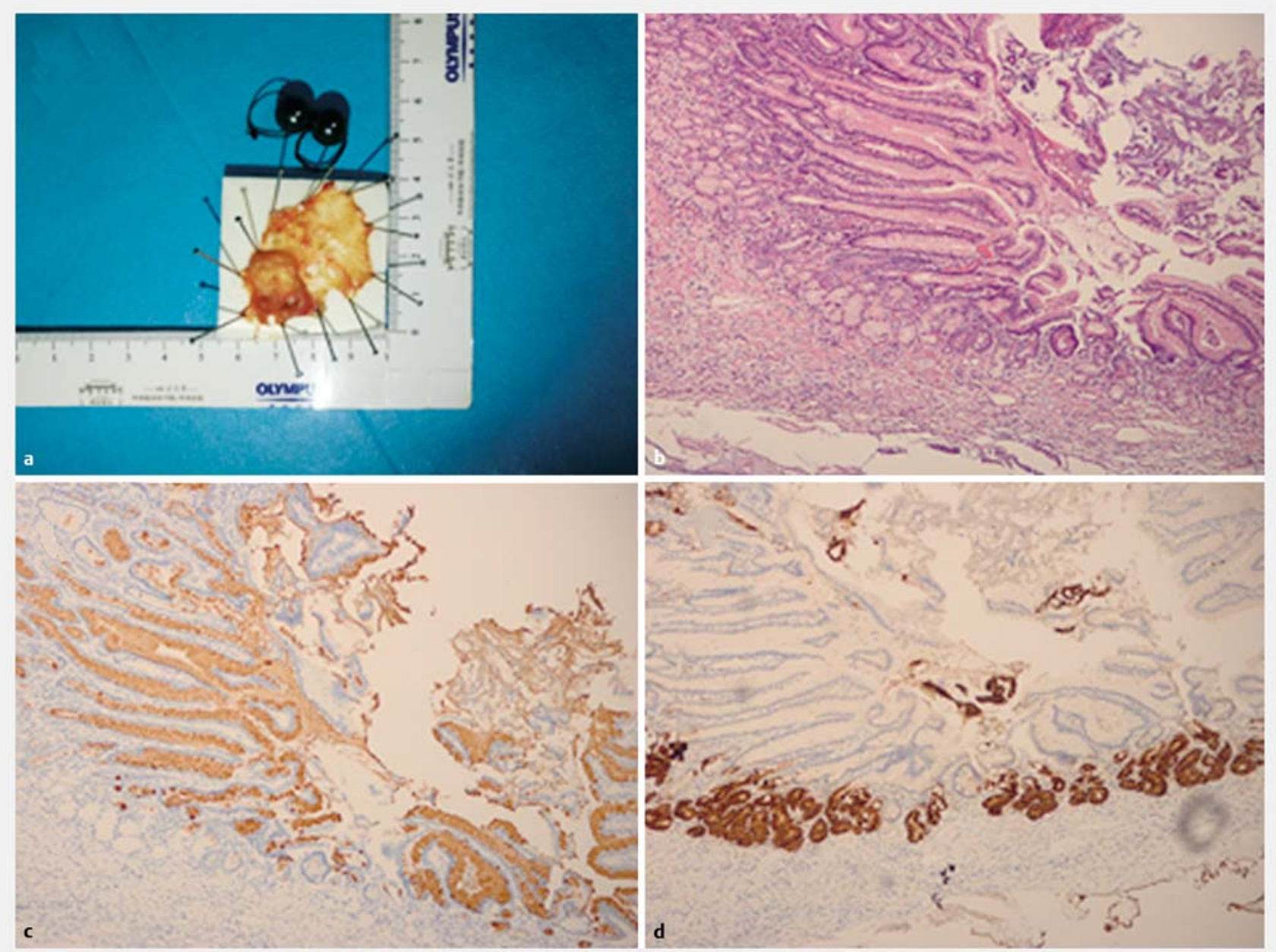

- Fig. 4 Specimen and pathological result. a The lesion after complete en bloc resection, with two magnetic beads. b Hematoxylin and eosin $(\mathrm{H} \& \mathrm{E})$ staining showed heterotopic gastric mucosa. $\mathbf{c}$ Immunohistochemical staining for MUC5AC was positive. $\mathbf{d}$ Immunohistochemical staining for MUC6 was positive.
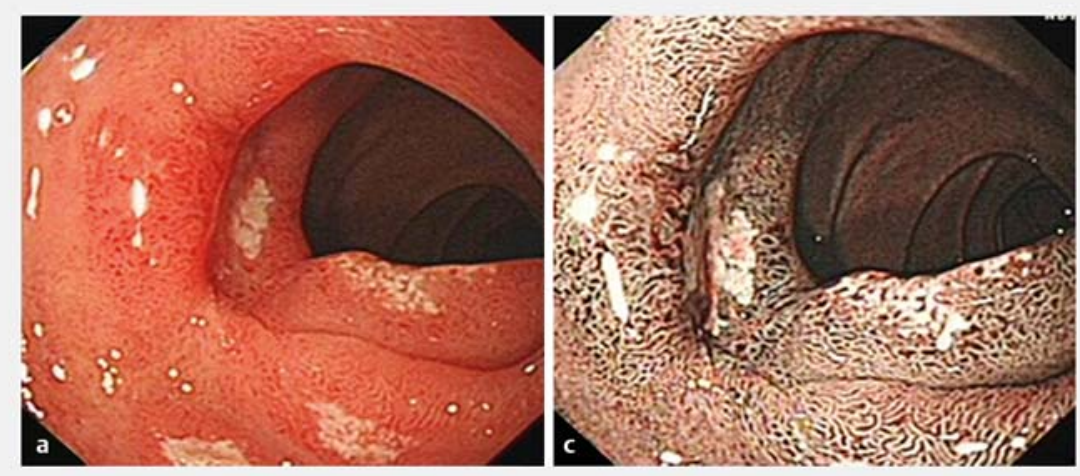

Bibliography

DOI https://doi.org/10.1055/a-0836-2545

Published online: 21.2 .2019

Endoscopy 2019; 51: E113-E115

(c) Georg Thieme Verlag KG

Stuttgart $\cdot$ New York

ISSN 0013-726X

Fig. 5 The ulcer bed after endoscopic submucosal dissection (ESD) seen at follow-up after 1 month: a white light; $\mathbf{b}$ narrow band imaging. 